Meta

Journal des traducteurs

Translators' Journal

\title{
La traduction médico-pharmaceutique
}

\section{Henri Van Hoof}

Volume 15, numéro 2, juin 1970

URI : https://id.erudit.org/iderudit/004545ar

DOI : https://doi.org/10.7202/004545ar

Aller au sommaire du numéro

Éditeur(s)

Les Presses de l'Université de Montréal

ISSN

0026-0452 (imprimé)

1492-1421 (numérique)

Découvrir la revue

Citer cet article

Van Hoof, H. (1970). La traduction médico-pharmaceutique. Meta, 15(2),

95-109. https://doi.org/10.7202/004545ar d'utilisation que vous pouvez consulter en ligne.

https://apropos.erudit.org/fr/usagers/politique-dutilisation/ 


\section{la \\ trsaduction}

\section{médico-pharmaceutingue*}

Pour la clarté de notre exposé, nous voudrions préciser d'emblée que deux grandes questions retiendront notre attention : 1) Quel est le champ de la traduction médico-pharmaceutique ? 2) Quels sont les problèmes de la traduction médico-pharmaceutique?

\section{CHAMP DE LA TRADUCTION MÉDICO-PHARMACEUTIQUE}

Pourquoi parler de traduction médico-pharmaceutique et non, tout simplement, de traduction médicale? Quelle est la nuance? L'introduction de la notion de pharmacie fait apparaître par la même occasion la notion d'industrie - l'industrie spécialisée dans la recherche et la fabrication de médicaments. Or, les problèmes de traduction qui se posent à ce genre d'industrie sont d'une variété et d'une ampleur qui dépassent encore celles, pourtant appréciables déjà, de la traduction médicale pure.

Pour mettre en évidence cette variété et cette ampleur, il suffit de suivre pas à pas l'évolution d'un médicament depuis sa conception jusqu'à sa mise à la disposition du corps médical. Deux grandes étapes marquent cette évolution : celle de la recherche et celle de la commercialisation. Au stade précommercial, la traduction intervient aux niveaux de la documentation médicale, de la synthèse chimique et de la fabrication galénique des recherches pharmacologiques, de l'expérimentation animale et humaine. Le traducteur se voit donc d'entrée confronté avec des problèmes chimiques - et cela signifie qu'il doit savoir ce qu'est une formule brute et une formule développée pour traduire ces termes respectivement par empirical formula et structural formula; avec des problèmes pharmacologiques, toxicologiques et tératologiques - et cela signifie qu'il doit s'y retrouver dans les

* Communication présentée au "Colloque sur le langage médical ", Liège, octobre 1969. 
innombrables batteries de tests utilisés en laboratoire s'il veut traduire correctement activité tropinique par atropine-like activity mais spasme acétylcholinique par acetylcholine-induced spasm; avec des problèmes d'expérimentation clinique - et cela signifie qu'il doit être au courant des dernières méthodes statistiques pour traduire avec à propos double-blind cross-over trial par essai en double anonymat avec permutations croisées et randomization par distribution au hasard; avec des problèmes technologiques, enfin - et cela signifie qu'il ne peut ignorer ce qu'est un procédé d'analyse, un mode opératoire, un procès-verbal de contrôle s'il veut s'éviter le ridicule de traduire le français dosage (d'une matière active) par l'anglais «dosage », là où il faut titration (of an active substance); ou le français tournesol par l'allemand «Sonnenblume», là où il s'agit manifestement du réactif Lackmus ; ou l'anglais fat flask par le français «gros flacon», là où il suffirait de dire ballon.

Arrive ensuite le stade de la commercialisation. La traduction intervient alors au niveau de la documentation commerciale, car il faut savoir à quoi s'en tenir sur les produits similaires de la concurrence; au niveau scientifique, car il faut publier dans les revues médicales ou présenter à des congrès les travaux réalisés chez l'animal et chez l'homme avec le produit nouveau; au niveau promotionnel, car il faut faire connaître ce médicament au corps médical du monde entier par le truchement de documents publicitaires appropriés : brochures, dépliants, modes d'emploi, etc. Ici encore, le traducteur aura l'occasion de montrer tout son savoir-faire. Non seulement sur le plan de la terminologie pure - car il doit évidemment savoir que l'anglais dosage traduit ici le français posologie et non «dosage »- mais aussi sur celui de la recherche d'équivalences dans le message promotionnel. Et l'on sait la difficulté que comporte la traduction de slogans tels que $X Y Z$ tablets take the strain and ease the pain (pour un myorelâchant) ou We made a package deal with Trichomonas and Candida (pour un traitement combiné des infections vaginales), ou encore, en partant du français $X Y Z$ fait le poids (pour un produit grossissant). Le sujet certes est tentant, mais sortirait du cadre de cet exposé. Il fournit, en tout cas, une preuve supplémentaire de l'extrême diversité des tâches qui attendent le traducteur médico-pharmaceutique. Son domaine d'activité étant ainsi défini dans ses grandes lignes, nous pouvons examiner un peu plus en détail quelques-uns des problèmes de sa pratique quotidienne.

\section{PROBLÈMES DE LA TRADUCTION MÉDICO-PHARMACEUTIQUE}

Les problèmes principaux peuvent se répartir en trois grands groupes selon qu'ils procèdent d'usages différents dans l'une ou l'autre des communautés linguistiques envisagées, de l'emploi de termes courants dans des acceptions médicales par l'une ou l'autre de ces communautés, ou de divergences terminologiques pures. Pour illustrer ces divers groupes nous nous bornerons ici à des exemples puisés dans les domaines anglais et français.

\subsection{Différences d'usage entre communautés linguistiques}

Un des grands reproches que l'on adresse fréquemment aux traductions scientifiques ou techniques est de ne pas faire corps avec le jargon de la spécialité en cause : on sent qu'elles ne sont pas l'œuvre d'un habitué de cette spécialité. 
De là la querelle d'écoles qui oppose les partisans de la traduction faite par des professionnels d'une branche déterminée et revue par des linguistes, à ceux de la traduction faite par des linguistes et revue par des spécialistes de cette discipline. Nous ne nous hasarderons pas à trancher ici ce différend fondamental. L'important est de constater que si, de fait, beaucoup de traductions prêtent le flanc à semblable critique c'est parce que leurs auteurs ignorent, méconnaissent ou négligent, d'une part, certaines tendances propres au génie respectif des domaines linguistiques en présence, et, d'autre part, certains usages propres aux divers langages spécialisés existant dans l'un et l'autre de ces domaines.

Parmi les principaux traits distinctifs de l'anglais et du français, rappelons la possibilité pour le lexique anglais de faire appel au fonds germanique et au fonds latin et sa propension pour le terme d'extraction populaire plutôt que savante, la plus grande facilité de dérivation et de composition de l'anglais, sa plus grande économie avec le recours fréquent à l'ellipse, à la substitution catégorielle, à la cinétisation des prépositions, etc. Ces caractères généraux ne sont pas escamotables par le simple fait que l'on passe de la langue courante à un quelconque langage technique. Bien au contraire, et dans le cas du jargon médical notamment, certains d'entre eux s'y trouvent même renforcés.

2.1.1. Le langage médical se fonde sur un lexique essentiellement savant : coagulation, cicatrisation, hydrothérapie, lordose, myopie, macrodactylie, acroparesthésie nocturne, xérodermie, etc. Pour un très grand nombre de ces termes, l'anglais possède, à côté du mot savant, un mot du fonds populaire qu'il préfère ou ne possède en tout et pour tout que ce dernier. En voici quelques exemples :

articulation

coagulation

cicatrisation

hydrothérapie

lordose

macrodactylie

myopie

acroparesthésie

glycémie

tétanos

thésaurismose

tophus articulation, joint

coagulation, clotting

cicatrization, scarring

hydrotherapy, water cure

lordosis, hollow back, saddle back

macrodactylia, giant finger

myopia, nearsightedness, shortsightedness

restless legs

glycemia, blood sugar

tetanus, lockjaw

thesaurismosis, storage disease, accumulation disease

tophus, chalkstone

On. notera que, dans les cas où un terme français possède lui aussi des synonymes, ceux-ci sont le plus souvent de formation savante également :

xérodermie, astéatose cutanée

hespéranopie, amblyopie crépusculaire

niphablepsie, ophtalmie des neiges

schistosomiase cutané, dermatite

cercarienne

anarthrie, aphasie motrice

sous-corticale xeroderma, paper skin, parchment skin hesperanopia, twilight blindness

niphablepsia, snow blindness

schistosome dermatitis, water itch, swimmer's itch

anarthria, jumbled speech 
Même la dérivation adjectivale est plus savante en français, alors que par la simple adjectivation d'un substantif adéquat, habituellement tiré du fonds populaire, l'anglais peut se dispenser de désinences :

papilles gustatives

glande sudoripare

cataracte thermogène

scissure simienne

infection hydrique

articulation orbiculaire

cellule falciforme

grossesse gémellaire

jumeaux univitellins

taste -buds »
sweat gland
heat cataract
ape fissure
water-borne infection
ball-and-socket joint
sickle cell
twin pregnancy
one-egg twins
seal-fin deformity
jaw « jerk
trigger area
heat "rash »

main phocomélique

réflexe massétérin

zone réflexogène

érythème calorique

heat $\ll$ rash $»$

Dans les cas où le français procède par simple suffixation pour former ses adjectifs et si cette possibilité existe aussi pour l'anglais, celui-ci inclinera malgré tout à procéder comme ci-dessus :

épithélium pavimenteux

granules pigmentaires

maladie sérique

embolie graisseuse

corps riziformes

cancer roentgenien

\author{
pavement epithelium \\ pigment granules \\ serum sickness \\ fat embolism \\ rice bodies \\ roentgen carcinoma
}

Même s'il emploie un véritable abjectif, l'anglais le choisira moins savant que le français :

langue dépapillée

goutte tophacée

tic de nutation

douleur térébrante

ferment lipolytique

bald tongue

chalky gout

nodding spasm

boring pain

fat-splitting enzyme

Les abjectifs composés, fréquents en médecine et toujours du type savant en français, suivent cette règle beaucoup moins souvent en anglais :

épithélioma baso-cellulaire

sarcome giganto-cellulaire

anastomose termino-latérale

basal cell carcinoma

giant cell sarcoma

luxation acromio-claviculaire

end-to-side anastomosis

barrière hémato-encéphalique

knocked-down shoulder

blood-brain barrier

Quelle est la portée de ces constatations sur le plan pratique de la traduction? Elle peut revêtir deux aspects selon que l'on part d'un original anglais ou français. Le traducteur francophone pourra s'étonner, du moins au début, de la profusion de termes descriptifs et, partant, moins ésotériques, qui émaillent les textes médicaux anglo-saxons. S'ils sont de nature à lui faciliter la compréhension, il 
devia toutefois s'en méfier au moment de les traduire, car même si le français possède un équivalent descriptif, celui-ci jurera le plus souvent avec le ton plus savant qu'attend l'utilisateur de la version française et suffira peut-être à faire classer le traducteur parmi les amateurs. Prenons par exemple les mots itch et bleeding, pour lesquels le français offre respectivement démangeaison et saignement. Ils paraissent à première vue bien inoffensifs, et pourtant il serait risible de traduire winter itch par « démangeaisons hivernales 》, car le médecin francophone attend prurit hivernal; de même, si l'on peut, à la rigueur traduire nose bleeding par saignement de nez, jamais on ne parlera de «saignement intestinal massif 》 pour massive intestinal bleeding, mais bien d'hémorragie intestinale massive. Inversement, placé devant un texte médical à rendre en anglais, le traducteur francophone devra pour le moins s'interroger sur l'opportunité qu'il y a de substituer aux termes savants ou à une partie d'entre eux des mots plus simples mais plus courants dans l'usage médical anglo-saxon.

2.1.2. Dans le même ordre d'idées, il convient de signaler les difficultés que recèle un usage différent des valeurs intellectuelle et affective d'un même terme. L'anglais médical n'hésite pas à appliquer à l'homme, à l'organisme humain, des mots que le génie français réserve à des notions exclusivement techniques. Nous n'en voulons pour exemple que des mots comme defect, defective, damage, etc.

Septal defects are a common congenital heart disease.

Many children have defective eyes.

Il est clair que « défaut » et « défectueux » n'ont pas leur place dans une traduction médicale adéquate. Dans le premier cas, il faudra recourir à malformation, anomalie, voire à vice (speech defect, vice de parole), lacune (defect of visual field, lacune du champ visuel filling defect, image lacunaire [en radiologie]) ou tout simplement affection (valvular defect, affection valvulaire). Dans le second cas, une modulation du type moyen-résultat s'impose : Beaucoup d'enfants ont une mauvaise vue.

Renal damage may also occur.

Potassium is released by the damaged red cells.

Many mothers become permanently damaged in health by evil birthconditions. Ici encore, «dommage », 《dégâts », "préjudice », "tort » pour le substantif, « endommager» pour le verbe ne conviendront pas au ton d'une traduction médicale. Sans doute, le français connaît «s'abimer la santé » ou «amocher quelqu'un ", mais on conviendra que ces expressions relèvent de niveaux de langage fort éloignés de notre objectif. Pour le substantif, nous aurons le choix entre lésion, atteinte, pour le verbe entre léser et atteindre.

2.1.3. L'adjectivation du substantif dont il a été question plus haut ne constitue qu"une application particulière au langage médical de la plus grande économie qu'autorise la langue anglaise grâce à la substitution catégorielle. Sa dérivation adjectivale et adverbiale plus facile est une autre source d'économie que le langage médical ne se fait faute d'exploiter. Voyons, par exemple, ce qu'il advient des paires culture/culture, skeleton/squelette.

Diagnosis was based on actual culture of brucellosis (nom). 
No diagnosis is possible before cultural methods are used (adjectif).

There were 22 cases of culturally proven brucellosis (adverbe).

Effusions from which Brucella has been cultured (verbe).

Certes, nous avons «culturel » en français, et rien ne s'opposerait à en faire "culturellement», mais il est douteux qu'un médecin francophone accepte sans sourciller des « méthodes culturelles de diagnostic» ou une «brucellose prouvée culturellement ». Quant à to culture, le français n'a pas la possibilité de procéder par simple verbalisation du substantif et devra se contenter d'une locution verbale construite sur un nom : faire une culture (sur bouillon).

The drug relieves spasm in skeletal muscles.

A skeletal survey showed a normal skull.

Encore une fois, nous possédons l'adjectif «squelettique » en français, mais il comporte une connotation tellement affective qu'il en devient inapplicable à un contexte technique : «examen squelettique » est tout aussi impossible que « muscles squelettiques» et nous devons recourir à un adjectif de relation pour traduire correctement par examen du squelette et muscles du squelette.

2.1.4. L'ellipse des articles défini et indéfini, des articulations, voire de mots significatifs est une autre démarche typique de l'anglais au point que certains auteurs - britanniques d'ailleurs - ont pu l'accuser de «déloyauté foncière » pour omettre souvent la partie essentielle d'une expression. Le langage médical anglo-saxon en fait un usage abondant.

After a coronary occlusion (the) cardiac muscle becomes either dead or injured.

One case necessitated (a) Ceasarean section.

(The) Patients were invited to co-operate in the study of the effects of prenylamine.

Grammaticalement, on pouvait attendre l'article défini devant cardiac dans la première phrase, l'article indéfini devant Caesarean dans la seconde. Quant à la troisième, il eût été normal de la commencer par l'article défini, car manifestement son absence ne traduit pas le partitif français des.

Haemoglobin sickle cell disease is characterized by emboli formation.

En l'absence d'articulations entre les quatre premiers termes de cette phrase, le traducteur pourra envisager plusieurs découpages : haemoglobin/sickle/cell/disease; haemoglobin/sickle cell/disease; haemoglobin sickle/cell disease; haemoglobin/ sickle cell disease. C'est sa compétence de la terminologie médicale qui lui fera rejeter en l'occurence les trois premiers car il s'agit en réalité d'une affection de l'hémoglobine, dans laquelle les globules rouges ou hématies prennent la forme d'un croissant ou d'une faucille (sickle). On traduira donc : L'hémoglobinopathie à hématies falciformes se caractérise par la formation d'embols.

Occlusive dressing technique patient instruction booklet.

Dans cet autre exemple, le nombre de termes multiplie les possibilités de découpage. Certes, quelques groupements s'éliminent d'eux-mêmes (technique patient), mais il en reste suffisamment pour s'y perdre si l'on ignore qu'il s'agit d'une technique de pansement nouvelle, le pansement occlusif. D'où la traduction : Brochure d'instruc- 
tions à l'usage $d u$ patient concernant la technique $d u$ pansement occlusif, dans laquelle nous soulignons les articulations exigées par le français.

By lowering the blood sugar the attacks of angina increased.

Erect and supine abdominal films showed no evidence of intestinal obstruction.

Pour traduire correctement ces deux phrases, le traducteur doit normalement savoir que dans chacune d'elles il y a ellipse d'un terme significatif. Dans la première manque level car il s'agit en réalité du blood sugar level ou taux de glycémie de sorte que la traduction deviendra : Une réduction du taux de la glycémie multiplia les crises angineuses. Dans la seconde, c'est la notion de position qui doit être retrouvée pour donner son sens à la traduction française, laquelle pourra dès lors s'énoncer ainsi : Des radiographies abdominales en position assise et couchée ne révéleront aucun signe d'obstruction intestinale.

Que retirerons-nous de ces diverses observations? En premier lieu, que la pratique de la traduction médicale - comme d'ailleurs de tout autre type de traduction technico-scientifique - ne dispense pas d'une bonne connaissance des problèmes de la traduction en général. Trop souvent l'on entend dans la bouche de candidats traducteurs des réflexions de ce genre : "Très peu pour moi les finesses de la traduction littéraire! Dans la traduction technique au moins, tout n'est qu'une question de terminologie et, pour cela, il y a les dictionnaires. » L'erroné de pareille conception n'en apparaîtra que mieux lorsque nous traiterons plus loin des divergences terminologiques.

En second lieu, que si la traduction vers le français posera parfois au traducteur certains problèmes de découpage ou d'interprétation par la servitude linguistique dans laquelle il se trouve d'expliciter les articulations ou autres mots dont peut se passer l'original anglais, c'est cependant surtout en traduisant vers l'anglais que le traducteur francophone devra s'efforcer de se libérer du cadre plus analytique de la phrase française.

\subsection{Termes courants et acceptions médicales}

Toute langue offre l'exemple de termes du domaine courant qui, par extension sémantique, trouvent des applications nouvelles dans des langages techniques. Le langage médical n'échappe pas à cette règle, et l'anglais moins que tout autre, en raison précisément de sa propension au terme populaire. La traduction de tels mots dans leurs acceptions spécialisées ne coule pas toujours de source et c'est le type même de difficulté sur lequel les dictionnaires sont généralement muets. Quelques exemples glanés dans la littérature nous montreront ce qu'il advient en français de mots tels que history, complaint, approach, gross, initial, discharge, course, discomfort, distress, ready for use, etc., ou ce que deviennent en anglais des mots comme urgent, grave, etc.

\section{History}

The best method of diagnosing drug-induced disease is still a carefully taken history.

Le mot history s'applique à la description de l'état du malade avant sa visite chez le médecin et devra se traduire soit par anamnèse, terme savant (ex. : rien dans l'anamnèse du patient ne permet de conclure à l'existence d'un ulcère), soit par 
passé (ex. : un patient avec un passé dyspeptique). Dans la phrase proposée, seule la première traduction s'intégrera parfaitement au contexte : La meilleure méthode de diagnostic d'une affection médicamenteuse reste encore l'anamnèse détaillée.

Approach

Hysterectomy can be performed by the abdominal or vaginal approach.

Il n'est pas rare d'entendre des francophones, manifestement sous l'influence de l'anglais, parler de l'« approche » d'un problème, de l'« approche » psychologique de la publicité, etc. Solution de facilité sur le plan de la traduction, sans aucun doute, et dont un contexte médical ne pourrait s'accommoder. En chirurgie notamment, comme c'est le cas dans la phrase proposée, approach devra se traduire par voie d'abord.

\section{Initial}

The initial dosage was two tablets daily.

L'adjectif anglais initial, indépendamment du fait qu'il est trop souvent rendu erronément par le français «initial», prend en médecine un sens bien précis qu'il faut traduire en français par d'attaque. La phrase proposée devient ainsi : La posologie d'attaque était de deux comprimés par jour. Initial dosage s'oppose ici à maintenance dosage, posologie d'entretien et à booster dose, dose de rappel.

Early, late

Early diagnosis is essential in carcinoma of the breast.

The early results of the operation were good.

The treatment of early breast carcinoma is still a matter of controversy.

Involvement of the bones is a late complication of brucellosis.

The late results of heart transplantation are impredictable.

D'une simplicité apparemment enfantine pour un traducteur, ces antonymes peuvent prendre bien des nuances dans un contexte médical. Dans notre premier exemple, early correspond manifestement au français précoce : Un diagnostic précoce est essentiel dans le cancer du sein.

Mais peut-on parler de «résultats précoces» dans la seconde phrase, ou de «cancer précoce» dans la troisième? Les traductions correctes seront ici, d'une part, immédiats : Les résultats immédiats (les suites immédiates) de l'opération, et early est alors synonyme de short-term; d'autre part primaire : Le traitement du cancer primaire du sein reste un sujet controversé, car il s'agit en l'occurrence d'une tumeur qui n'a pas encore formé de métastases, et early est alors l'équivalent de primary.

De même si l'on peut envisager de traduire le premier late par tardif : Les lésions osseuses constituent une complication tardive de la brucellose, la solution ne vaut pas pour le second; ici late est synonyme de long-term et devra se rendre par éloigné : Les suites éloignées de la transplantation cardiaque sont imprévisibles. 


\section{Course}

There was no response to a course of penicillin injections.

The course of brucellosis is usually protracted.

De la comparaison de ces deux phrases il ressort nettement que le mot course est pris dans deux acceptions différentes. Dans la première, il faudra traduire par cure ou série : Une cure de piqûres de pénicilline demeura sans effet. Dans la seconde par cours ou évolution : L'évolution de la brucellose est habituellement longue.

\section{Discharge}

At the time of discharge the E.E.G. showed no significant abnormality.

In the second patient there was profuse nasal discharge.

Dans les deux phrases, également, l'anglais discharge couvre deux notions médicales différentes. Dans la première, il s'agit d'une sortie d'hôpital et l'on pourrait - après avoir fait apparaître le patient que le texte anglais escamote - envisager la traduction : Au moment où le patient put quitter l'hôpital, son E.E.G. n'accusait aucune anomalie significative. Dans la seconde phrase, il s'agit d'un écoulement et la traduction deviendra : Chez le deuxième patient, l'écoulement nasal était abondant, ou, en recourant au terme savant : la rhinorrhée était abondante.

\section{Tender, tenderness}

The lymph glands in the right groin were enlarged, firm and tender.

There was moderate distention of the abdomen and tenderness in the pericumbilical region.

Dans la première phrase, firm et tender se contrediraient dès l'instant où l'on se risquerait à traduire ce dernier mot par «tendre»; il doit donc s'agir d'autre chose. L'acception médicale de tender est celle de douloureux à la pression, de sorte que les deux exemples cités se liraient en français : Les ganglions inguinaux gauches étaient gonflés, durs, et douloureux à la pression, et : Il y avait peu de distension abdominale et de la douleur à la pression dans la région péri-ombilicale.

La liste pourrait s'allonger encore, mais telle quelle son pouvoir d'illustration devrait suffire. Avant de clôturer le sujet, cependant, nous retiendrons un dernier terme, français celui-là, l'adjectif urgent. Même le profane sait ce qu'on appelle une urgence en médecine et que l'anglais traduit par emergency. Mais essayer de rendre abdomen urgent par « emergency abdomen» ou par «urgent abdomen» aboutirait à un effet risible pour le médecin anglo-saxon, pour qui seul acute abdomen existe.

\subsection{Divergences terminologiques pures}

Si nous parlons maintenant de problèmes de terminologie pure, c'est pour les distinguer de ceux que nous avons abordés jusqu'ici et que l'on peut considérer cornme régis en grande partie encore par certains traits fondamentaux du génie de la langue. Les difficultés d'ordre terminologique pur sont nombreuses, et comme il serait présomptueux d'en faire ici l'inventaire complet, nous tenterons d'en apporter la preuve par la sélection de quelques points particulièrement représentatifs, à savoir, les désinences, les synonymes, les éponymes et les abréviations. 
2.3.1. Problèmes de désinences - Nous avons vu que, fréquemment, l'anglais n'est pas obligé ou n'éprouve pas le besoin de recourir à des suffixes pour sa dérivation adjectivale. Lorsqu'il le fait, on serait tenté de croire que des terminaisons aussi simples que -AL (cervical, mental, seminal), -ARY (coronary, biliary, urinary), -IC (colic, epidemic, trochanteric) et autres se laisseront calquer sans plus par les désinences françaises correspondantes : -AL(E), -AIRE, -IQUE, etc.

De fait, si l'on considère les innombrables paires qui répondent au schéma ...AL/...AL(E) :

catarrhal/catarrhal (e)

cervical/cervical(e)

costal/costal(e)

hemorrhoïdal/hémorroïdal(e)

renal/rénal(e)

temporal/temporal( $e$ )

on pourrait conclure que tous les adjectifs anglais en - $\mathrm{AL}$ se traduiront automatiquement par une forme française en - $\mathrm{AL}(\mathrm{E})$. Il suffira pourtant de quelques sondages dans la littérature médicale courante pour faire apparaître immédiatement une bonne douzaine d'autres possibilités :

-AIRE : Dental/dentaire, placental/placentaire

-ATIF : germinal/germinatif (épithélium, par ex.)

-E : portal/porte (veine, par ex.)

-É : sacral/sacré, larval/larvé

-ÉAL : subungual/sous-unguéal

-EUX : anginal/angineux

-IEN : carpal/carpien, retinal/rétinien

-IFÈRE : seminal/séminifère

-INE : palatal/palatine

-IQUE : aortal/aortique, streptococcal/streptococcique

-ONNIÈRE : mental/mentonnière (artère, par ex.)

-UE : vagal/vague

-ULAIRE: appendical/appendiculaire

Sans doute les dictionnaires spécialisés fourniront-ils la réponse adéquate dans la plupart des cas, mais encore faudra-t-il déterminer la forme française appropriée au contexte, dans les cas où un même adjectif anglais en -AL possède plusieurs équivalents en français. C'est ainsi que larval epilepsy devra se traduire par épilepsie larvée, alors que larval disease peut être une affection larvaire. Mental condition ne peut évidemment se traduire autrement que par état mental, mais la mental artery des Anglo-Saxons est notre artère mentonnière. On traduira seminal cells par cellules séminales, mais seminal ducts par canalicules séminifères. Et si portal circulation appelle normalement circulation portale, il faut cependant rendre portal vein par veine porte.

De même, sur la base de nombreuses paires axillary/axillaire biliary/biliaire coronary/coronaire maxillary/maxillaire pulmonary/pulmonaire la désinence -ARY semblerait devoir toujours se traduire par -AIRE. Il lui arrive cependant de revêtir d'autres formes telles que :

-ARIEN : coronary/coronarien

-EUX : biliary/bilieux, urinary/urineux 
qui sont le plus souvent des doublets de la forme en -AIRE. La difficulté provient alors de la confusion possible entre ces doublets : si urinary tract et urinary calculus entraînent logiquement les traductions appareil urinaire et calcul urinaire, il faut pourtant rendre urinary abscess par abcès urineux; biliary calculus et biliary fistule se traduisent respectivement par calcul biliaire et fistule biliaire, mais à biliary fever correspond fièvre bilieuse; et si coronary vessel devient vaisseau coronaire, il faudra pourtant parler d'onde coronarienne pour coronary wave.

Quant à la désinence -IC, en dehors de son correspondant évident -IQUE, comme dans :

antihistaminic/antihistaminique limbic/limbique

colic/colique tympanic/tympanique

elle n'offre pas moins d'une demi-douzaine de variantes :

-AIRE : embryonic/embryonnaire

$-\mathrm{E}:$ androgenic/androgène, hypochronic/hypochrome

-IAQUE : manic/maniaque

-IE : epidemic/épidémie

-ïDIEN : allantoic/allantoïdien (liquide, par ex.)

-IN : masseteric/massétérin

-OGÈNE : antigalactic/antigalactogène

Lorsque plusieurs formes françaises correspondent à la terminaison -IC d'un même mot anglais, comme pour trochanteric, qui peut appeler trochantérien et trochantérique, ou epidemic qui peut engendrer épidémie et épidémique, il faudra veiller à les appliquer judicieusement. Dans le second cas, il sera certes assez facile de ne pas confondre substantif et adjectif; dans le premier, l'expérience de la terminologie médicale fera traduire trochanteric line par ligne intertrochantérique et tendinotrochanteric ligament par ligament tendinotrochantérien.

2.3.2. Problèmes de synonymie - Le langage médical constitue peut-être le plus beau cas de prolifération synonymique que l'on puisse imaginer. Innombrables sont les concepts désignés par plusieurs expressions, en principe équivalentes, mais différentes selon qu'elles procèdent d'une vision anatomique, chimique, pathogénique, toponymique, historique ou tout simplement descriptive des choses. Maladie de Heine-Médin est la dénomination historique donnée à une atteinte de la substance grise de la moelle épinière, connue aussi sous les appellations clinique de paralysie spinale infantile et anatomique de polyomyélite antérieure aiguë. De même, on a employé indistinctement les termes d'anémie splénique infectieuse ou pseudoleucémique, de leishmaniose splénique infantile, de lymphadénie splénique des nourrissons, de pseudoleucémie infantile, de fièvre épidémique d'Assam, pour le kala-azar infantile; de fièvre sudorale, folle ou ondulante et de fièvre méditerranéenne ou de Malte pour la mélitococcie ou brucellose affection que les Britanniques désignaient encore par Cyprus fever, Gibraltar fever et Rock fever. Car les synonymes ne sont pas toujours superposables d'une langue à l'autre. En anglais, infectious granuloma, malignant granuloma ou lymphoma, lymphadenoma, anemia lymphatica, pseudoleukemia sont autant d'équivalents pour ce que le médecin francophone appelle lymphogranulomatose maligne et que son confrère anglo-saxon connaît surtout sous le nom de Hodgkin's disease. De même, la polyarthrite déformante, que le Français identifie encore au rhumatisme 
chronique déformant, est surtout connue sous le vocable de polyarthrite chronique évolutive pour lequel l'O. M. S. suggère à présent d'adopter polyarthrite rhumatö̈de et dont l'équivalent usuel anglais est rheumatoid arthritis, en dépit des synonymes arthritis deformans, atrophic arthritis, chronic infectious arthritis et proliferative arthritis. Toujours dans le domaine rhumatismal, la rheumatic fever des Anglo-Saxons, avec ses synonymes acute articular rheumatism et polyarthritis rheumatica, a pour équivalent le plus courant en français, malgré l'existence des termes fièvre rhumatismale et polyarthrite aiguë fébrile, la dénomination de rhumatisme articulaire aigu (souvent abrégé en $\mathrm{R}$. A. A.) et, davantage encore peut-être, de maladie de Bouilland. Or, le médecin anglo-saxon connaît aussi l'appellation de Bouilland's disease, mais elle couvre pour lui la notion de bacterial (ou infective) endocarditis. Nous reviendrons d'ailleurs plus loin sur la confusion engendrée par les éponymes.

Il ne faudrait pas croire que cette pléthore synonymique se limite aux noms de maladies. L'observation vaut tout autant pour les notions anatomiques, physiologiques, techniques et autres. Le blastoderme des francophones peut appeler en anglais les équivalents blastoderm, blastodermic disk, blastodisk, blastodermic ou primitive ectoderm, germinal membrane; le bulbe duodénal peut se traduire par duodenal bulb, sans doute, mais aussi par duodenal cap, pyloric cap, bishop's cap; ce que nous appelons en français tomographie ou stratigraphie peut devenir pour les Anglo-Saxons, outre tomography et stratigraphy, analytical roentgenography, body sectional roentgenography, laminography, planigraphy, radiotomy, sectional roentgenography, vertigraphy. En partant de l'anglais, le phénomène s'observe pour des termes même très simples, avec la restriction toutefois que, parmi les équivalents français possibles, le choix sera dicté par le contexte. Ainsi, le mot fold pourra devoir se traduire par pli, repli, cul-de-sac, ligament, sillon, valvule, crête, vallée, bourrelet, échancrure, éminence, mais sans que ces termes soient toujours interchangeables. Si gluteal fold peut engendrer à la fois pli et sillon fessier, et sublingual fold à la fois repli et éminence sublingual(e), dans la plupart des cas, cependant, le terme anglais n'appellera qu'une seule traduction correcte pour fold :

nail fold

neural fold

ciliary folds

palatine folds

lacrimal fold

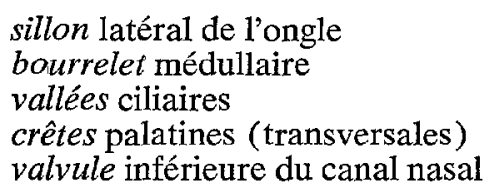

Un phénomène analogue s'observe pour groove (sillon, fente, gouttière, coulisse, rainure, espace), line (ligne, liseré, crête, bandelette, repli, strie), seam (bordure, liséré, marge, ourlet, raphé), process (processus, apophyse, tubercule, bourgeon, éminence, épine, appendice, prolongement) et quantité d'autres termes, que l'on pourrait d'ailleurs considérer comme autant d'exemples supplémentaires d'emprunts à la langue courante dans des acceptions médicales.

2.3.3. Problèmes d'éponymie - Le problème des éponymes représente un aspect particulier et un obstacle supplémentaire du labyrinthe synonymique médical. Les éponymes médicaux sont de deux types, selon que le nom propre a donné nais- 
sance à un nom commun (addisonism/addisonisme) ou est demeuré nom propre qualifiant une maladie, un symptôme, une technique, un appareil déterminé (Banti's disease/maladie de Banti). Déjà les noms communs nous réservent quelques surprises, car la forme qu'ils adoptent dans une langue n'existe pas toujours ou n'est pas nécessairement la même dans l'autre langue. Le basedowisme des Français est le basedowiform disease des Anglo-Saxons; l'endothéliome kupfférien des uns est le kupferoma des autres; la fossette gassérienne n'est connue des Anglo-Saxons que sous le nom de trigeminal impression; tandis qu'à l'inverse leur fallopian pregnancy devient une grossesse tubaire et que leur franklinism désige ce que les Français connaissent sous le nom de bain statique. La darsonvalisation est l'application thérapeutique des courants à haute fréquence découverte par le physicien français d'Arsonval, mais le traducteur non initié soupçonnera-t-il que c'est par ce terme qu'il faut rendre teslaization (méthode identique mise au point par Tesla, de New York) dans un texte américain?

Les noms propres employés tels quels ne font que rendre le problème plus complexe, tout d'abord parce qu'ils sont beaucoup plus nombreux, ensuite parce que sur le plan de la traduction ils ne répondent à aucune règle. Un terme anglais construit sur un éponyme peut très bien avoir un équivalent français sans éponyme et vice versa, ou à un éponyme anglais peut très bien correspondre un autre éponyme en français, ou l'éponyme peut être identique dans les deux langues mais se rapporter à un nom différent pour le même concept ou à deux concepts différents, etc. Prenons par exemple la maladie de Paget, et son pendant anglais Paget's disease : la correspondance est tout à fait illusoire, car par ce vocable l'Anglo-Saxon désigne l'ostéite déformante et le véritable équivalent éponymique français est maladie osseuse de Paget tandis que la maladie de Paget des francophones devra se traduire par Paget's disease of the nipple. De même, la maladie de Recklinghausen, synonyme en français de polyfibromatose névrocutanée pigmentaire. Mais il existe aussi la maladie osseuse de Recklinghausen, qui désigne alors l'ostéite fibrokystique. Or, en anglais, Recklinghausen's disease couvre à la fois la neurofibromatose et l'ostéite fibrokystique.

Le casse-tête s'aggrave encore lorsque plusieurs éponymes se greffent sur une même affection, comme dans le cas du goitre exophtalmique appelé par les uns maladie de Basedow, par les autres maladie de Graves ou de Marsh, et que les Anglo-Saxons connaissent encore sous les noms de Flajani's disease et Parry's disease, comme aussi le syndrome d'Adair-Dighton que les Français nomment encore maladie d'Apert ou syndrome de Lobstein-Cornazon, mais pour lequel l'anglais possède, à côté de Adair-Dighton syndrome, des équivalents éponymiques différents, Eddowes' syndrome et Spurway-Eddowes syndrome. Lorsqu'on songe, d'autre part, que l'opération de Finsterer (procédé de gastrectomie partielle le plus usité en France) s'appelle aussi opération de Hofmeister, qu'elle représente une variante du procédé de Polya, lui-même synonyme d'opération de Reichel et correspond pour le chirurgien d'expression anglaise à Billroth's operation II (à ne pas confondre avec Billroth's operation I qui traduit l'opération de Péan), on ne peut que conseiller la plus extrême circonspection au traducteur placé devant de tels termes. 
2.3.4. Problèmes d'abréviations - Tout jargon scientifique et technique se forge ses abréviations propres, et le langage médical n'échappe pas à cette règle. Le procédé, s'il n'est pas pour faciliter la tâche du traducteur, trouve cependant sa justification dans le fait qu'un auteur francophone pourrait hésiter à répéter en toutes lettres et à longueur de texte des mots tels que polyarthrite chronique évolutive ou concentration inhibitrice minimale, par exemple ; il préférera peut-être utiliser les abréviations $P$. C. E. et $C . I$. M., qu'il sait connues de tous ses confrères spécialisés. Au traducteur de ne point ignorer que, dans la branche du rhumatisme, P. C. E. doit se rendre en anglais par R. A. (rheumatoid arthritis) et que la C. I. M. répond, en antibiothérapie, à la $M . I$. C. (minimum inhibitory concentration) des Anglo-Saxons.

Toute une série d'abréviations devraient normalement faire partie du bagage du traducteur et ne pas poser de problèmes, soit parce qu'elles ne changent pas de forme en anglais, comme $E$. E. G. pour électro-encéphalogramme, E. C. G. pour électrocardiagramme, $I$. $M$. pour intramusculaire, $I$. $V$. pour intraveineux, anti-B. $K$. pour anti-tuberculeux (où $B . K$. remplace Bacille de Koch), etc., soit qu'elles se substituent à des notions tellement fondamentales que même un changement de forme du français à l'anglais n'en peut empêcher l'identification, telles L. C. R. pour liquide céphalo-rachidien (C.S.F. pour cerebrospinal fluid), $V . S$. pour vitesse de sédimentation, ( $S . R$. ou $E . S . R$. pour (erythrocyte) sedimentation rate), V.B.P. pour voie biliaire principale (C.B.D. pour common bile duct), $S$. N.C. pour système nerveux central (C.N.S. pour central nervous system), etc. Mais, à ce stade déjà, la confusion guette le traducteur, car si $S$. $R$. est l'abréviation de sedimentation rate, elle est aussi celle de sigma reaction; de même, $C$. $F$. abrège parfois non seulement cerebrospinal fluid, mais encore chest foot (en électrocardiographie et, il est vrai, d'habitude sans les points).

Souvent, le français reprend telles quelles les abréviations créées par l'anglais. Ainsi l'hormone adrénocorticotrope ou corticostimuline est-elle sans doute mieux connue sous l'abréviation A.C.T. H. (de l'anglais Adreno-Cortico-Tropic Hormone); de même, l'hormone folliculo-stimulante ou gonadostimuline a pour abréviation F.S. H. (de l'anglais Follicle-Stimulating Hormone) et l'hormone lutéinisante L. H. (de l'anglais Luteinizing Hormone) ou I. C.S.H. (de l'anglais Interstitial-Cell Stimulating Hormone). À l'inverse, l'anglais emprunte parfois des abréviations au français telle $B . C$. G. pour bacille de Calmette-Guérin ou Calmette-Guérin Bacillus. Les noms de substances thérapeutiques sont eux aussi fréquemment abrégés au départ de l'anglais : D. O.C.A. pour l'acétate de désoxycorticostérone (de l'anglais Des-Oxy-Corticosterone Acetate), P. A. S. pour l'acide para-amino-salicylique (de l'anglais Para-Amino-Salicylic Acid), B. A. L. pour les 2,3-dimercaptopropanol (de l'anglais British Anti-Lewisite), L. S. D. pour l'amide de l'acide lysergique (de l'anglais Lysergic Acid Diethylamide), etc. Enfin, dans le domaine des techniques de laboratoire des études pharmacologiques, biochimiques, cliniques, etc., le traducteur se heurtera sans cesse à des abréviations qui sont, avant tout, des sigles de travail :

$D L(50)$ pour dose létale $(50)$

$D E(50)$ pour dose efficace $(50)$

$L D(50)$ pour lethal dose (median)

$E D(50)$ pour effective dose (median) 
S. L.I. pour stimulation lumineuse intermittente

$V E M$ pour volume expiratoire

$V E M S$ pour volume expiratoire maximum seconde
I. L.S. pour intermittent light stimulation

$F E V$ pour forced expiratory volume $F E V$ pour forced expiratory volume for one second

parfois sans abréviation équivalente dans l'une ou l'autre langue :

formule leucocytaire

electrochoc

réaction de fixation du complément
W. B.C. pour white blood count

E.S.T. pour electric shock therapy

C. $F$. $T$. pour complement fixation test intravenous urography

On objectera peut-être que les dictionnaires spécialisés sont faits pour s'en servir et que la solution à la plupart des problèmes soulevés doit bien s'y trouver. Nous sommes d'accord jusqu'à un certain point. Mais, dans un domaine aussi fluctuant que celui de la médecine, où chaque jour apporte sa moisson de découvertes, de techniques nouvelles, de perfectionnements dans les méthodes d'investigation et de traitement, il est normal qu'un ouvrage lexicographique soit périmé avant même sa sortie de presse. Il est impossible au compilateur de serrer l'actualité d'assez près. Or, les textes que le traducteur médico-pharmaceutique se voit confier sortent, le plus souvent, tout droit de l'actualité. Comme dans tout domaine spécialisé, il doit être son propre lexicographe et, pour cela, fréquenter assidument la littérature spécialisée, c'est-à-dire connaître ses sources d'information.

HenRI VAN HoOF 